\title{
Lanthanide Sensitizers for Large Anti-Stokes Shift Near-Infrared-to-Visible Triplet-Triplet Annihilation Photon Upconversion
}

Natalia Kiseleva, ${ }^{\dagger}$ Pariya Nazari, ${ }^{\dagger}$ Carolin Dee, ${ }^{\ddagger}$ Dmitry Busko, ${ }^{\dagger}$ Bryce S. Richards, ${ }^{\dagger, \nabla}$ Michael Seitz, ${ }^{\ddagger}$ Ian A. Howard, ${ }^{*, \dagger, \nabla}$ Andrey Turshatov, ${ }^{\dagger}$

†Institute of Microstructure Technology, Karlsruhe Institute of Technology, Hermann-von-HelmholtzPlatz 1, 76344 Eggenstein-Leopoldshafen, Germany

‡Institute of Inorganic Chemistry, University of Tübingen, Auf der Morgenstelle 18, 72076 Tübingen, Germany

${ }^{\nabla}$ Light Technology Institute, Karlsruhe Institute of Technology, Engesserstrasse 13, 76131 Karlsruhe, Germany

\section{Experimental Section}

\section{Chemicals}

Rubrene (99.99\%), thenoyltrifluoroacetone, $\mathrm{YbCl}_{3} \cdot 6 \mathrm{H}_{2} \mathrm{O}$ (99.9\% Yb), 1,10-phenanthroline, and deuterated tetrahydrofurane ([D8]-THF, 99.5\%D) were purchased in from Sigma-Aldrich (Merck). Ethanol for the synthesis of $\mathbf{Y b}-\mathbf{L}$ was HPLC-grade.

\section{Synthesis of Yb-L}

Yb-L was synthesized according to a slightly altered literature procedure. ${ }^{1}$

In a round-bottom-flask, thenoyltrifluoroacetone (667 mg, $3.00 \mathrm{mmol}, 3.0$ equivs.) was suspended in EtOH (10 mL) and solid 1,10-phenanthroline (180 mg, $1.00 \mathrm{mmol}, 1.0$ equiv.) was added in portions. The mixture was heated to $75^{\circ} \mathrm{C}$ (bath temperature) until a clear solution was obtained. The solution was allowed to cool to $30^{\circ} \mathrm{C}$ (bath temperature) and an aqueous solution of $\mathrm{NaOH}(1 \mathrm{M}, 3.00 \mathrm{mmol}, 3.00 \mathrm{~mL}$, 3.0 equivs.) was added dropwise. The solution was stirred at the same temperature for 30 minutes before a solution of $\mathrm{YbCl}_{3} \cdot 6 \quad \mathrm{H}_{2} \mathrm{O}$ (388 mg, $1.00 \mathrm{mmol}, 1.0$ equiv.) in water (7 mL) was added under efficient stirring. After complete addition, the mixture was heated back up to $60^{\circ} \mathrm{C}$ (bath temperature) and stirred at this temperature for $3.5 \mathrm{~h}$. The suspension was allowed to cool to ambient temperature under stirring, the precipitate was collected on a Büchner funnel and washed with a minimum of ice-cold EtOH (ca. $1 \mathrm{~mL}$ ). After drying the solid under reduced pressure for $5 \mathrm{~h}$, the crude product was recrystallized from $\mathrm{CHCl}_{3}$ (dissolve hot in a minimum of solvent, crystallization upon cooling to $4^{\circ} \mathrm{C}$ ), collected on a Büchner 
funnel, washed with ice-cold $\mathrm{CHCl}_{3}$ (ca. $1 \mathrm{~mL}$ ), and dried in vacuum to yield the title compound as pale yellow solid (722 mg, $71 \%$ ). The analytical data were in agreement with previously reported data. ${ }^{1-2}$

${ }^{1} \mathrm{H}$ NMR (400 MHz, CD ${ }_{2} \mathrm{Cl}_{2}$ ): $\delta$ = 18.8 (s, $2 \mathrm{H}$ ), 18.1 (d, $\left.J=6.3 \mathrm{~Hz}, 2 \mathrm{H}\right), 13.1$ (d, $\left.J=6.5 \mathrm{~Hz}, 2 \mathrm{H}\right), 9.45$ (s, 2 H), 6.67 (d, $J=5.0$ Hz, 3 H), 5.46 (s, 3 H), 2.51 (s, 3 H), 11.2 (s, 3 H) ppm.

\section{Sample preparation}

The solution of Yb-L (concentration 0.1 M) and rubrene (concentration $1 \mathrm{mM}$ ) in [D8]-THF was prepared inside a $\mathrm{N}_{2}$-filled glovebox (GS GLOVEBOX Systemtechnik GmbH) with concentration of oxygen $0.1 \mathrm{ppm}$. The solution was placed in $1 \mathrm{~mm}$ quartz cuvette (Hellma Analytics) and sealed inside the glovebox. The sample was stored in the glovebox between measurements.

\section{Optical characterization}

UV/VIS absorption spectra were recorded on a spectrophotometer (Lambda 950, PerkinElmer). The absorption spectra of all solutions were measured in a $1 \mathrm{~mm}$ quartz cuvette (Hellma Analytics) against the cuvette with THF as a reference. For photoluminescence (PL) of $\mathrm{Yb}^{3+}$, the cuvette was excited with $980 \mathrm{~nm}$ continuous-wave (CW) laser diode (Thorlabs, L980P200) mounted on a temperature-controlled mount (Thorlabs, TCLDM9) and driven using a laser diode controller (Thorlabs, ITC4001). The $\mathrm{Yb}^{3+} \mathrm{PL}$ was captured using a spectrometer (Avantes, AvaSpec - ULS2048 × 64TEC). The upconversion (UC) emission was excited with the $980 \mathrm{~nm}$ laser diode (Thorlabs, L980P200). The power of the beam and beam shape was measured with a power meter (Thorlabs, PM320E) and beam profiler (Thorlabs, BP209 - IR/M), respectively. For characterisation of the intensity dependency of UC emission the laser beam was attenuated with motorized variable filter wheel (Thorlabs, NDC - 100C - 2). The UC excitation spectra was measured with earlier described experimental procedure. ${ }^{3}$ Detailed description of measurements of PL lifetime and QY Uc has been also reported earlier. ${ }^{4}$ 


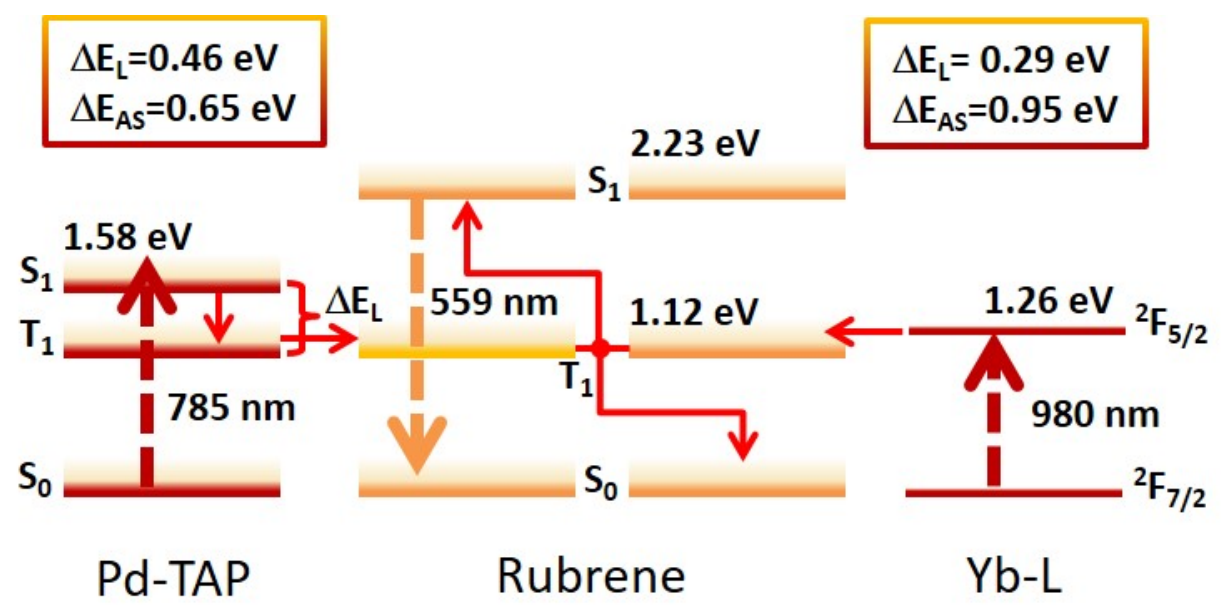

Figure S1. Scheme of TTA-UC with palladium tetraanthraporphyrin (Pd - TAP) ${ }^{5}$ and $\mathrm{Yb}-\mathrm{L}$ as sensitizer and rubrene as emitter. The scheme displays the amplitude of anti-Stokes shift for both Pd-TPA $(0.65 \mathrm{eV})$ and $\mathrm{Yb}-\mathrm{L}(0.95 \mathrm{eV})$ sensitizers. The energy losses $\left(\Delta \mathrm{E}_{\mathrm{L}}=0.46 \mathrm{eV}\right)$ due to internal conversion and intersystem crossing is much higher for Pd-TPA as compared with Yb-L $(0.29 \mathrm{eV})$.

To prove the lack of the direct excitation of rubrene with $980 \mathrm{~nm}$ laser we performed experiments with the exact same excitation conditions for which we observed UC for two other solutions as well:

1) Solution of rubrene in $\left[\mathrm{D}_{8}\right]$-THF

2) Solution of new $\mathrm{Yb}^{3+}$-based sensitizer (Figure S2) mixed with rubrene in [D8]-THF.

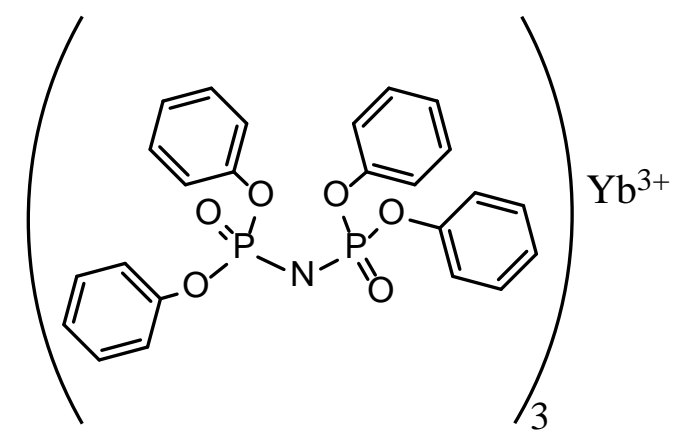

Figure S2. Chemical structure of $\mathrm{Yb}^{3+}$ complex which doesn't demonstrate TTA-UC luminescence.

We were not able to detect visible luminescence of rubrene under $980 \mathrm{~nm}$ excitation (under maximal available intensity of the laser) for both solutions. 


\section{References}

(1) Freund, C.; Porzio, W.; Giovanella, U.; Vignali, F.; Pasini, M.; Destri, S.; Mech, A.; Di Pietro, S.; Di Bari, L.; Mineo, P. Thiophene Based Europium $\beta$-Diketonate Complexes: Effect of the Ligand Structure on the Emission Quantum Yield Inorg. Chem. 2011, 50, 5417-5429.

(2) Doffek, C.; Seitz, M. The Radiative Lifetime in Near-IR Luminescent Ytterbium Cryptates - The Key to Extremely High Quantum Yields. Angew. Chem. Int. Ed. 2015, 54, 9719-9721.

(3) Meijer, M. S.; Rojas-Gutierrez, P. A.; Busko, D.; Howard, I. A.; Frenzel, F.; Würth, C.; ReschGenger, U.; Richards, B. S.; Turshatov, A.; Capobianco, J. A.; Bonnet, S. Absolute upconversion quantum yields of blue-emitting $\mathrm{LiYF}_{4}: \mathrm{Yb}^{3+}, \mathrm{Tm}^{3+}$ upconverting nanoparticles. Phys. Chem. Chem. Phys. 2018, 20, 22556-22562.

(4) Joseph, R. E.; Jiménez, C.; Hudry, D.; Gao, G.; Busko, D.; Biner, D.; Turshatov, A.; Krämer, K.; Richards, B. S.; Howard, I. A. Critical Power Density: A Metric To Compare the Excitation Power Density Dependence of Photon Upconversion in Different Inorganic Host Materials. J. Phys. Chem. A 2019, 123, 6799-6811

(5) Yakutkin, V.; Aleshchenkov, S.; Chernov, S.; Miteva, T.; Nelles, G.; Cheprakov, A.; Baluschev, S. Towards the IR Limit of the Triplet-Triplet Annihilation-Supported Up-Conversion: Tetraanthraporphyrin. Chem. - Eur. J. 2008, 14, 9846-9850. 